\title{
Commentary
}

\section{Role of Cystatin C in Neuroprotection and Its Therapeutic Implications}

\author{
Luciano D’Adamio \\ From the Department of Microbiology \& Immunology, Albert \\ Einstein College of Medicine, New York, New York
}

Earlier studies have suggested that cystatin C (CysC) is a target for intervention in neurological disorders because its expression increases in response to brain insults, in human neurological disorders, and in animal models of neurodegenerative states. However, these studies did not clarify whether CysC up-regulation is a pathogenic factor in neurodegenerative disorders or whether it represents a neuroprotective compensatory response of the organisms aimed to prevent progression of the disease. Recent reports have shown that CysC protects neuronal cells from toxic insults induced by a variety of stimuli in vitro, ${ }^{1}$ suggesting that therapeutic approaches aimed to either increase the levels of $\mathrm{Cys} C$ or to mimic its action could be beneficial in neurological disorders. However, the in vivo relevance of these findings remained unclear. In this issue of the journal, the article by Kaur et $\mathrm{al}^{2}$ presents for the first time results supporting the in vivo efficacy of CysC as a neuroprotective protein.

Unverricht-Lundborg disease (progressive myoclonic epilepsy type 1 [EPM1]), caused by autosomal recessive loss-of-function mutations in the gene encoding cystatin $\mathrm{B}$ (CysB, also known as Stefin B), ${ }^{3}$ is the most common cause of progressive myoclonus epilepsy worldwide. ${ }^{3-5}$ EPM1 is relatively frequent in the western Mediterranean region but is most prevalent around the Baltic Sea. It is a neurodegenerative disorder with age of onset from 6 to 16 years, characterized by stimulus-sensitive myoclonus and tonic-clonic epileptic seizures. Ataxia, loss of coordination, and tremor develop several years after the onset of the disease. Individuals with EPM1 are mentally alert but show depression and mild decline in intellectual performance over time. ${ }^{6}$ Cerebellar atrophy and motor cortex degeneration are largely responsible for the loss in equilibrium, correlating with the motor symptoms of the disease. ${ }^{7}$ The diagnosis of EPM1 is confirmed by identifying disease-causing mutations in the CysB gene.

The CysBKO mouse is an established EPM1 model with clinical symptoms similar to those of patients with EPM1. In CysBKO mice, cerebellar granule cells ${ }^{8}$ and neurons within the hippocampal formation and entorhinal cortex degenerate, ${ }^{9}$ suggesting that $\mathrm{Cys} B$ is a physiological regulator of normal survival of certain neuronal populations and providing a direct explanation for the causes of neuronal degeneration in EPM1. Thus, understanding the mechanisms by which loss of CysB leads to neuronal degeneration in mice will shed light on the biochemical and cellular mechanisms leading to EPM1, thereby providing a rational for therapeutic targets. The efficacy of therapeutic strategies can indeed be tested in this particular model for EPM1.

Cystatins have been implicated in the processes of neuronal degeneration and repair of the nervous system. ${ }^{10}$ Both CysC and CysB are potent, reversible inhibitors of most of the currently known cathepsins. Cathepsins are proteinases required for housekeeping functions during protein turnover that differ in structure, substrate specificities, and biochemical characteristics. ${ }^{11}$ The cystatins effectively block these catalytically active proteinases; thus, the extent of proteolytic activity at any given time and location is the result of a balance between active proteases and physiological inhibitors. Any abnormal variation in levels and/or activity of either proteases or their endogenous inhibitors will result in deregulation of these enzymatic activities causing pathological changes (for review, see ref. 12). Uncontrolled proteolysis as a result of imbalance between active proteases and their endogenous inhibitors has been associated with neuronal cell death in different neuronal diseases, including brain tumors, stroke, some forms of epilepsy, Alzheimer's disease, and neurological autoimmune diseases. ${ }^{12}$ Indeed, recent data suggest that reduced proteolytic activity, caused by mutations in the familial Alzheimer's disease gene PS1, inhibit autophagy and promote neuronal degeneration. ${ }^{13}$

In other instances, pathogenic mutations may cause neurodegeneration by increasing proteolytic activities. EPM1 seems to be a prime example of this situation, because it is caused by loss of function mutations in the protease inhibitor CysB gene, and data suggest that increased proteolysis by lysosomal cathepsins is respon-

Accepted for publication August 26, 2010.

Address reprint requests to Luciano D'Adamio, M.D., Ph.D., luciano. dadamio@einstein.yu.edu. 
sible for phenotypic characteristics of EPM1. ${ }^{14-16}$ This hypothesis is supported by evidence that deletion of cathepsin $\mathrm{B}$ in CysBKO mice resulted in a reduction in the amount of cerebellar granule cell apoptosis, depending on mouse age. ${ }^{15}$

Cathepsins are activated at low pH $(\sim 4.2)$ and are mainly active in lysosomes, although some reports show that cathepsins can also be found in extracellular compartments. CysB, a member of the cystatin family 1 of cysteine protease inhibitors, is diffusely distributed in the cytoplasm of all cell types ${ }^{15}$ and is present, albeit at low levels, in body fluids. ${ }^{17}$ Kaur et $\mathrm{al}^{2}$ show that intracellular CysB is mainly present in lysosomes, further supporting the model implicating $\mathrm{CysB}$ as a physiological inhibitor of activity of cathepsins. Cys $\mathrm{C}$ belongs to the type 2 family of cystatins and is targeted extracellularly via the secretory pathway. ${ }^{12}$ Accordingly, CysC is found in all mammalian body fluids and tissues (for review, see ref. 18). However, extracellular CysC is in part internalized via endocytosis. Through the endocytic compartment, CysC is targeted to the lysosomal system where it can function as an inhibitor of cathepsin activities. ${ }^{12}$

Based on earlier evidence, ${ }^{1}$ Kaur et $\mathrm{al}^{2}$ asked whether CysC exerts a neuroprotective activity in vivo as a result of its ability to inhibit the activity of lysosomal cathepsins. First, the authors found a pronounced increase in CysC expression level in CysBKO mice compared with that in wild-type mice. As previously stated, enhanced CysC expression occurs in human patients with epilepsy and animal models of neurodegenerative conditions, as well as in response to injury (for review, see ref. 12). Thus, up-regulation of CysC expression in EPM1 mice could represent an intrinsic neuroprotective mechanism that counteracts progression of the disease, an attempt to rescue neurons by inhibiting the apoptosis-promoting actions of cathepsins in various vesicular domains, in the cytosol, and in the extracellular space. This hypothesis was tested by Kaur et $\mathrm{al}^{2}$ by crossing CysBKO mice with either mice overexpressing CysC or CysCKO mice. They demonstrate that $\mathrm{CysC}$ overexpression has widespread positive effects on the pathologies observed in CysBKO mice. CysBKO/CysC transgenic mice present reduced cerebellar granule cell loss and gliosis compared with CysBKO animals. As a result, CysC improved motor coordination and reduced the extent of imbalance present in EMP1 mice. On the contrary, ablation of CysC expression had an opposite effect: cortical and cerebellar neurodegeneration, cerebellar atrophy, and other pathologies are more pronounced in CysKO/CysBKO mice than in CysBKO animals. Although the worsening of disease in CysKO/CysBKO mice underscores the role of endogenous CysC in containing the EMP1 pathology, the beneficial effects of CysC in preventing anatomical (cerebellar atrophy, neuronal loss, and gliosis) and clinical (motor coordination) pathologies suggest that Cys C could be an efficacious therapeutic agent to alleviate behavioral and physiological deficits present in EPM1.

Increased mRNA, protein, and enzymatic activity levels of the two lysosomal enzymes cathepsins B and D in the cortex and cerebellum of CysBKO mice suggest that CysB deficiency causes lysosomal dysfunction and cell damage, resulting in neurodegeneration. ${ }^{2}$ This impairment was reversed by Cys $\mathrm{C}$ overexpression as demonstrated by decreased cathepsin B and cathepsin D activities in the brains of CysBKO/CysC mice compared with those of CysBKO mice. Whereas it is convincingly demonstrated that CysC effectively counteracts the pathologies in CysBKO mice, none of the pathologies studied recovered to wild-type control levels by overexpression of CysC in CysBKO mice. This partial effect could be explained by a toxic outcome of high $\mathrm{Cys} C$ levels in the brains of CysBKO/CysC mice, because localized very high Cys Concentrations may be neurotoxic, as suggested by the observation that CysC injection into rat hippocampus caused neuronal degeneration in the site of the injection. ${ }^{19}$ In CysBKO/CysC mice, the combination of $\mathrm{Cys} C$ overexpression with the endogenously elevated CysC levels present in CysBKO animals may lead to localized toxic concentrations of CysC. On the other hand, it is possible that CysC cannot completely compensate for CysB loss of function. CysB is mainly localized in lysosomes, where cathepsins are active. CysC is instead secreted, and endocytosis of extracellular CysC transports only a fraction of CysC to lysosomes. Therefore, the levels of lysosomal CysC may be insufficient to normalize cathepsin activity in CysBKO/CysC transgenic mice. Thus, strategies to more efficiently target $\mathrm{Cys} C$ to lysosomes may improve the effectiveness of CysC (or mimics) in the prevention of neurodegeneration in EPM1.

Patients with EPM1 require lifetime clinical follow-ups, extensive drug-based therapy, and ample rehabilitation. Anti-epileptic treatments are currently used as therapeutic interventions in patients with EPM1. Valproic acid, the first drug of choice, diminishes myoclonus and the frequency of generalized seizures. Clonazepam and highdose piracetam are used to treat myoclonus, and levetiracetam seems to be effective for both myoclonus and generalized seizures. There are a number of agents that intensify the clinical course of EPM1, such as phenytoin, aggravating the associated neurological symptoms or even accelerating cerebellar degeneration. Sodium channel blockers (carbamazepine and oxcarbazepine) and GABAergic drugs (tiagabine and vigabatrin) as well as gabapentin and pregabalin aggravate myoclonus and myoclonic seizures. Almost all types of anti-epileptic agents aggravate associated neurological symptoms, accelerating cerebellar degeneration (for review, see ref. 6). Long-term side effects of anti-epileptic drugs can be avoided if restorative strategies are used.

The findings by Kaur et $\mathrm{al}^{2}$ indicate that $\mathrm{CysC}$ is neuroprotective in EPM1, imply that CysC, or a CysC-mimicking analog, if used at optimum concentrations could be a therapeutic candidate with a potential of preventing neurodegeneration. In addition, this work has strong implications for other age-related neurodegenerative disorders in which lysosomal dysfunction plays a considerable role. ${ }^{20}$ Currently, treatment of many neurodegenerative disorders involves only symptomatic interventions. CysC may gain clinical relevance for such neurological disorders, e.g., Alzheimer's disease, for which no preventive or curative drugs exist. 


\section{References}

1. Tizon B, Ribe EM, Mi W, Troy CM, Levy E: Cystatin C protects neuronal cells from amyloid-beta-induced toxicity. J Alzheimers Dis 2010, 19:885-894

2. Kaur G, Mohan P, Pawlik M, DeRosa S, Fajiculay J, Che S, Grubbs A, Ginsberg SD, Nixon RA, Levy E: Cystatin $C$ rescues degenerating neurons in a cystatin B-knockout mouse model of progressive myoclonus epilepsy. Am J Pathol 2010, 177:2256-2267

3. Pennacchio LA, Lehesjoki AE, Stone NE, Willour VL, Virtaneva K, Miao J, D'Amato E, Ramirez L, Faham M, Koskiniemi M, Warrington JA, Norio R, de la Chapelle A, Cox DR, Myers RM: Mutations in the gene encoding cystatin B in progressive myoclonus epilepsy (EPM1). Science 1996, 271:1731-1734

4. Lafreniere RG, Rochefort DL, Chretien N, Rommens JM, Cochius JI, Kalviainen R, Nousiainen U, Patry G, Farrell K, Soderfeldt B, Federico A, Hale BR, Cossio OH, Sorensen T, Pouliot MA, Kmiec T, Uldall P, Janszky J, Pranzatelli MR, Andermann F, Andermann E, Rouleau GA: Unstable insertion in the $5^{\prime}$ flanking region of the cystatin $B$ gene is the most common mutation in progressive myoclonus epilepsy type 1 , EPM1. Nat Genet 1997, 15:298-302

5. Lalioti MD, Scott HS, Buresi C, Rossier C, Bottani A, Morris MA, Malafosse A, Antonarakis SE: Dodecamer repeat expansion in cystatin B gene in progressive myoclonus epilepsy. Nature 1997, 386:847-851

6. Kälviäinen R, Khyuppenen J, Koskenkorva P, Eriksson K, Vanninen R, Mervaala E: Clinical picture of EPM1-Unverricht-Lundborg disease. Epilepsia 2008, 49:549-556

7. Koskenkorva P, Khyuppenen J, Niskanen E, Kononen M, Bendel P Mervaala E, Lehesjoki AE, Kalviainen R, Vanninen R: Motor cortex and thalamic atrophy in Unverricht-Lundborg disease: voxel-based morphometric study. Neurology 2009, 73:606-611

8. Pennacchio LA, Bouley DM, Higgins KM, Scott MP, Noebels JL, Myers RM: Progressive ataxia, myoclonic epilepsy and cerebellar apoptosis in cystatin B-deficient mice. Nat Genet 1998, 20:251-258

9. Shannon P, Pennacchio LA, Houseweart MK, Minassian BA, Myers RM: Neuropathological changes in a mouse model of progressive myoclonus epilepsy: cystatin B deficiency and Unverricht-Lundborg disease. J Neuropathol Exp Neurol 2002, 61:1085-1091

10. Zerovnik $E$ : The emerging role of cystatins in Alzheimer's disease. Bioessays 2009, 31:597-599

11. Turk B, Turk D, Turk V: Lysosomal cysteine proteases: more than scavengers. Biochim Biophys Acta 2000, 1477:98-111

12. Tizon B, Levy E: Protease inhibitors and their involvement in neurological disorders. Neurochemistry and Molecular Neurobiology: Neural Protein Metabolism and Function, ed 3. Edited by A Lajtha, NL Banik. New York, Springer, 2006, pp 591-624

13. Lee JH, Yu WH, Kumar A, Lee S, Mohan PS, Peterhoff CM, Wolfe DM Martinez-Vicente M, Massey AC, Sovak G, Uchiyama Y, Westaway D, Cuervo AM, Nixon RA: Lysosomal proteolysis and autophagy require presenilin 1 and are disrupted by Alzheimer-related PS1 mutations. Cell 2010, 141:1146-1158

14. Rinne R, Saukko P, Jarvinen M, Lehesjoki AE: Reduced cystatin B activity correlates with enhanced cathepsin activity in progressive myoclonus epilepsy. Ann Med 2002, 34:380-385

15. Houseweart MK, Pennacchio LA, Vilaythong A, Peters C, Noebels JL, Myers RM: Cathepsin B but not cathepsins $L$ or $S$ contributes to the pathogenesis of Unverricht-Lundborg progressive myoclonus epilepsy (EPM1). J Neurobiol 2003, 56:315-327

16. Lieuallen K, Pennacchio LA, Park M, Myers RM, Lennon GG: Cystatin B-deficient mice have increased expression of apoptosis and glial activation genes. Hum Mol Genet 2001, 10:1867-1871

17. Franceschetti S, Sancini G, Buzzi A, Zucchini S, Paradiso B, Magnaghi G, Frassoni C, Chikhladze M, Avanzini G, Simonato M: A pathogenetic hypothesis of Unverricht-Lundborg disease onset and progression. Neurobiol Dis 2007, 25:675-685

18. Levy E, Jaskolski M, Grubb A: The role of cystatin C in cerebral amyloid angiopathy and stroke: cell biology and animal models. Brain Pathol 2006, 16:60-70

19. Nagai A, Ryu JK, Kobayash S, Kim SU: Cystatin C induces neuronal cell death in vivo. Ann NY Acad Sci 2002, 977:315-321

20. Nixon RA, Yang DS, Lee JH: Neurodegenerative lysosomal disorders: a continuum from development to late age. Autophagy 2008, 4:590-599 